\title{
Shear velocity as the function of frequency in heavy oils
}

\author{
De-hua Han and Jiajin Liu, Rock Physics Lab, UH; \\ Micheal Batzle, Colorado School of Mine
}

\section{Summary}

A new frequency model for shear velocity of heavy oils is discussed based on the measured data in our lab. Havriliak and Negami (HN) model is suggested to describe the frequency dispersion of heavy oils.

\section{Introduction}

The velocity behavior in heavy oil depends on oil phase (Han et. al, 2006). As shown in Figure 1, heavy oil in the liquid phase at a higher temperature, $\mathrm{S}$-wave velocity is negligible and $\mathrm{P}$-wave velocity shows negligible frequency dependent, similar as conventional liquid oil. We have found a threshold of viscosity for the liquid phase of heavy oil is $\sim<800 \mathrm{cp}$, which called as the liquid point (Han, et al., 2006). As a heavy oil in the glass solid phase (viscosity $>10^{15} \mathrm{cp}$ ) there are both $\mathrm{P}$ - and S-wave velocities which have negligible frequency dependent dispersion, similar as an elastic solid. However, there is transition zone called quasi-solid phase for heavy oils. In this phase, viscosity of heavy oil is high enough to bear the shear stress. In addition, both shear rigidity and bulk modulus of the heavy oil are frequency dependent: high at ultrasonic, but low at sonic and seismic (Han et. Al., 2005). Clearly, we need to quantify the velocity dispersion behavior.

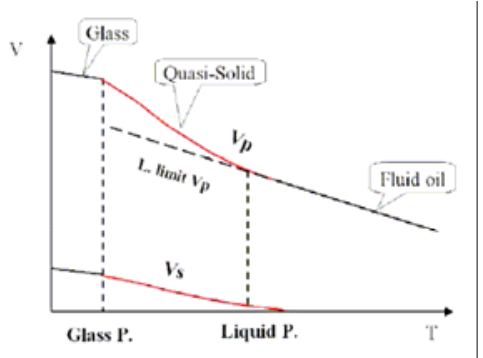

Fig. 1 Velocities of heavy oil in phase transition

Direct measure of velocity dispersion is always difficult if it is not possible. There are few data set measured at both low (a few Hz) and ultrasonic frequencies (Behera et al, 2006), which provide important indication how shear rigidity of an ultra-heavy oil (API $=-5$ ) depends on temperature and frequencies. The majority of velocity data is measured at ultrasonic frequencies $(0.5$ or $1 \mathrm{MHz})$ in different temperatures. We have to use those data to develop and calibrate a frequency dependent velocity model. First, we have developed a heavy oil model based on the Maxwell visco-elastic model and Beggs and Robinson (1975) viscosity model (Han, et. Al., 2005). The model shows a reasonable match with the shear velocity data measured in ultrasonic. But we found the both the Maxwell and viscosity models did not work well at low frequencies. Here we are going to introduce a new velocity dispersion model with applying a new viscosity model.

\section{Data set creation}

As we known, the effect of frequency on velocities can be equivalent to the temperature effect in the $\omega \tau$ domain in which $\omega$ is the angular frequency and $\tau$ is the relaxation time. The relaxation time depends on heavy oil viscosity, which is controlled mainly by temperature. Therefore, we can scale the ultrasonic data from temperature domain through a proper viscosity model into $\omega \tau$ domain, and to find the relationship between the shear velocity and frequency.

The following steps are used to transfer the data set of the shear velocity $\mathrm{Vs}_{\mathrm{i}}$ (and shear modulus $\mu_{\mathrm{i}}$ ) versus temperatures $\left(\mathrm{T}_{\mathrm{i}}\right)$ for a sample into a new set in the $(\omega \tau)$ domain.

1. Calculate the glass point that is the temperature at which the viscosity of the sample is $10^{15}$ centi-Poise according to its API degree using the specified viscosity model. We have tested several viscosity models such as Beggs-Robinson, De Ghetto (for heavy and extra heavy oil respectively), Bennison, etc. All the models seem underestimate the glass point of heavy oil (overestimate viscosity at low temperature less than $-10^{\circ} \mathrm{C}$ ). However, we have not find a proper viscosity model for heavy oil yet. We known that API is not a sole parameter to dominate viscosity, composition of heavy oil may also be important. Currently, we only concern API effect on viscosity and use the De Ghetto (for extra heavy) model in this work.

2. Estimate the shear velocity $\mathrm{Vs}_{\mathrm{g}}$ at the glass point. And calculate the shear modulus $\mu_{\mathrm{g}}$ at the glass point according to $\mathrm{Vs}_{\mathrm{g}}$. $\mu_{\mathrm{g}}$ will be taken as the reference modulus at the ultrasonic frequency $(1 \mathrm{MHz})$.

3. Estimate the viscosity $\eta_{i}$ for each temperature $T_{i}$ to get the data set of shear velocity versus viscosity.

4. Calculate relaxation time $\tau_{\mathrm{i}}$ for each viscosity using the formula,

$$
\tau_{\mathrm{i}}(\mathrm{s})=\frac{\eta_{\mathrm{i}}(\mathrm{cP})}{\mu_{\mathrm{g}}(\mathrm{GPa})}
$$

where $\tau_{\mathrm{i}}$ is the relaxation time, $\eta_{\mathrm{i}}$ is the viscosity and $\mu_{\mathrm{g}}$ is the reference shear modulus.

5. Calculate the normalized shear modulus $G_{i}$

$$
\mathrm{G}_{\mathrm{i}}=\frac{\mu_{\mathrm{i}}}{\mu_{\mathrm{g}}},
$$


where $\mu_{\mathrm{i}}$ is shear modulus for each viscosity. $G_{\mathrm{i}}$ is normalized shear modulus with the reference shear modulus $\mu_{\mathrm{g}}$.

We have created a data set of normalized shear moduli $G_{i}$ versus $(\omega \tau)$ for each sample with different API gravity (figure 2). The next step is to find an empirical model to fit the data, the model then can be used to predict the shear modulus and velocity at different frequencies.

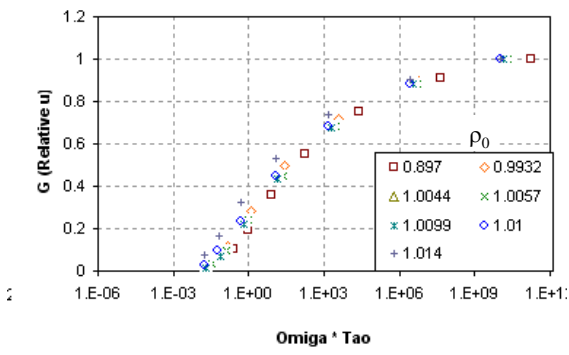

Fig. 2 Created data set of normalized shear modulus versus $\omega \tau$ for various APIs.

\section{Frequency model}

The Maxwell Model is a basic frequency model for viscoelastic liquids (figure 3).

$$
\mathrm{G}(\omega)=\mathrm{G}^{\prime}(\omega)+i \mathrm{G}^{\prime \prime}(\omega)=\frac{i \omega \tau}{1+i \omega \tau}
$$

where, $G(\omega)$ is the relative shear modulus as the function of frequency; $G^{\prime}(\omega)$ and $G^{\prime \prime}(\omega)$ are the real part and imaginary part of the shear modulus respectively.

The Maxwell Model has been modified by many researchers such as the Cole-Cole (19xx) or the Caputo (20xx) Model (figure 3). It is found that these models are exchangeable and can be written in the general format.

$$
G(\omega)=G^{\prime}(\omega)+i G^{\prime \prime}(\omega)=\frac{(i \omega \tau)^{\alpha-\beta}}{1+(i \omega \tau)^{\alpha-\beta}} \quad 0 \leq \beta<\alpha \leq 1
$$

The model will transform to the Maxwell Model when the parameters $\alpha=1$ and $\beta=0$, and to the Cole-Cole Model if $\alpha=1$ and is Caputo Model if $\beta=0$.

It is clear that the Cole-Cole Model is exactly the same as the Caputo Model if $\alpha=1-\beta$. Therefore, we can use one parameter $\alpha$ to describe the Maxwell, the Cole-Cole, or the Caputo Models. The figure 3 shows the complex shear modulus (relative) as the function of $\omega \tau$. Its norm is shown in the bottom figure. The real part (left) and imaginary part (right) is in the upper figure respectively. The parameter $\alpha$ changes the slope of frequency dependence, but the real part of the normalized shear modulus G' is always equal to 0.5 at $(\omega \tau)=1$ (upper left of figure 3 ). The frequency dependence shows symmetric to the center point $\mathrm{G}^{\prime}=$ 0.5 .
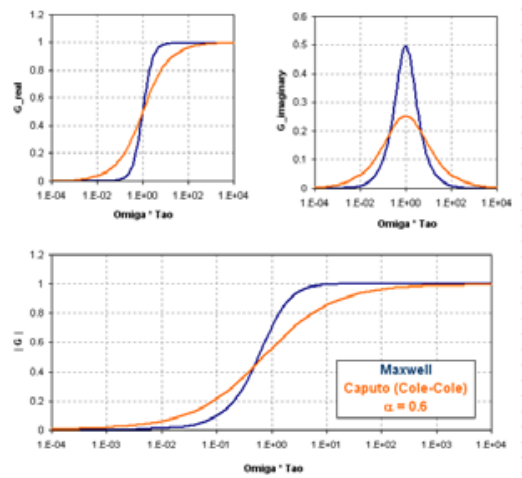

Fig. 3 Maxwell and Caputo (Cole-Cole) models

We have fit data using Caputo Model. Regression result shows that optimum value of the parameter $\alpha=0.19$ in figure 4. The model cannot fit the data well and shows that trend of the normalized shear modulus is not symmetric to the center point. It means the heavy oil may not be a viscoelastic liquid. It is necessary to use other models to describe the frequency dependent property of heavy oil.

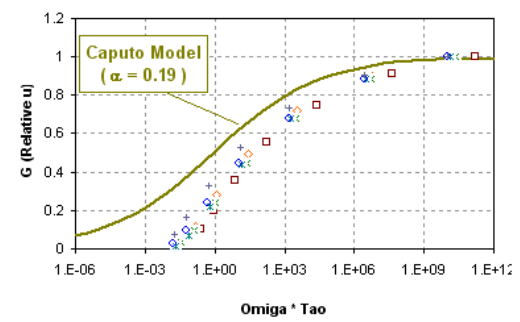

Fig. 4 Caputo model cannot fit the measured data well

Davidson and Cole (1951) developed a modified model (CD Model) in order to encompass the "skew" in the Cole-Cole arcs. Incorporation of this concept resulted in the following empirical equations:

$$
\begin{aligned}
G(\omega) & =G^{\prime}(\omega)+i G^{\prime \prime}(\omega)=1-\frac{1}{[1+(i \omega \tau)]^{\gamma}} \\
G^{\prime}(\omega) & =[\cos (\phi)]^{\gamma} \cos (\phi \gamma) \\
G^{\prime \prime}(\omega) & =[\cos (\phi)]^{\gamma} \sin (\phi \gamma) \\
\phi & =\operatorname{arctg}(\omega \tau)
\end{aligned}
$$

The CD model is shown in figure 5. Its norm is shown in the bottom figure and the real part (left) and imaginary part (right) is shown respectively in the upper figure. The model is not symmetric to the center point and the 
curve bends more in the higher part of $\omega \tau$ that is similar to the Caputo model in Figure 4.

Havriliak and Negami (1967) devised a two-parameter model that combined the concepts of both the Cole-Cole and CD models. The resulting Havriliak-Negami (HN) model is denoted as equation below in which the values of $\alpha$ and $\gamma$ retain their original meanings from the Cole-Cole and CD models.
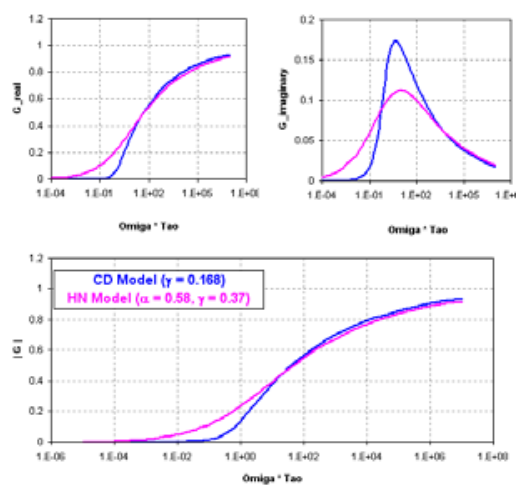

Fig. 5 CD (Cole-Davidson) and HN (HavriliakNegami) Models

$$
\begin{aligned}
& G(\omega)=G^{\prime}(\omega)+i G^{\prime \prime}(\omega)=1-\frac{1}{\left[1+(i \omega \tau)^{1-\alpha}\right]^{\gamma}} \\
& G^{\prime}(\omega)=1-R^{-\gamma / 2} \cos (\theta \gamma) \\
& G^{\prime \prime}(\omega)=R^{-\gamma / 2} \sin (\theta \gamma) \\
& R=\left[1+(\omega \tau)^{1-\alpha} \sin \left(\frac{\pi \alpha}{2}\right)\right]^{2}+\left[(\omega \tau)^{1-\alpha} \cos \left(\frac{\pi \alpha}{2}\right)\right]^{2} \\
& \theta=\operatorname{arctg}\left[\frac{(\omega \tau)^{1-\alpha} \cos \left(\frac{\pi \alpha}{2}\right)}{1+(\omega \tau)^{1-\alpha} \sin \left(\frac{\pi \alpha}{2}\right)}\right]
\end{aligned}
$$

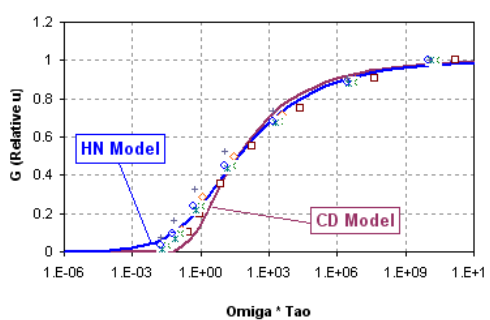

Fig. 6 Data fitting with CD and HN models

We fit data with both the CD model and the HN model. Regression results show the optimum value $\alpha=0.168$ for the CD model and $\alpha=0.58$ and $\gamma=0.37$ for the HN model. The figure 6 shows that the data fitting for both CD Model and HN model respectively. It can be seen in the figure that the CD model may be a little over straightened in the low frequencies to fit our data and the HN Model can fit the data better. Therefore, the HN Model is used to describe the frequency effect on shear modulus.

\section{Application}

The HN Model with the parameters obtained from our data ( $\alpha=0.58$ and $\gamma=0.37$ ) can be used to predict the frequency effect on shear velocity of the heavy oil with different API. Figure 7 shows an example for the heavy oil with API $=9.38\left(\rho_{0}=1.0044 \mathrm{~g} / \mathrm{cc}\right)$ that shear velocity versus frequency with different temperatures (left) and versus temperature with different frequencies (right). Data show consistency with the model. The model shows a complicated relationship of shear velocity dependence of frequencies and temperature for heavy oil. Shear velocity tends to be zero at temperature higher than the liquid point, which is frequency dependent (increases with increasing frequencies). In this condition, heavy oil is in liquid phase. Shear velocity tends to be independent of frequencies at temperature lower than the glass point. For heavy oil in the quasisolid phase with temperature between liquid point and glass point, shear velocity is dispersed from zero to the value of heavy oil at glass point. Without mention of frequency, shear velocity data of heavy oil in those temperature range is meaningless. Figure shows that for temperature ranged from -10 to $40{ }^{\circ} \mathrm{C}$ shear velocity of heavy oil in ultrasonic, logging and seismic frequency can be significantly different.
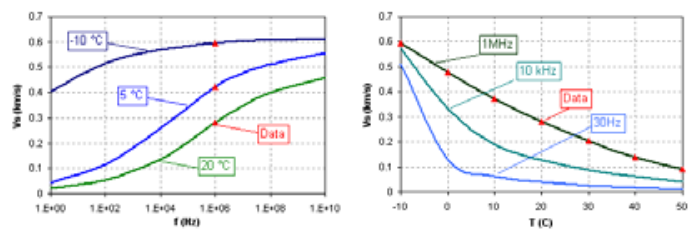

Fig. 7 Model calculated shear velocity versus frequency (left) and temperature (right) for the sample with $\rho_{0}=$ $1.0044 \mathrm{~g} / \mathrm{cc}$.

\section{Conclusion}

Correlation of shear modulus in frequency-viscosity domain to that in viscosity-temperature domain provides a transformation of temperature dependent shear modulus to frequency dependent one. We have used this method to developed shear modulus model as function of frequency for heavy oil. Measured data suggest that the Maxwell model did not work well with heavy oil. Currently, NH model in combination to the De Ghetto viscosity mode is the best to fit heavy oil data. 


\section{EDITED REFERENCES}

Note: This reference list is a copy-edited version of the reference list submitted by the author. Reference lists for the 2007 SEG Technical Program Expanded Abstracts have been copy edited so that references provided with the online metadata for each paper will achieve a high degree of linking to cited sources that appear on the Web.

\section{REFERENCES}

Batzle M., R. Hofmann, and D. Han, 2006, Heavy oils—seismic properties: The Leading Edge, 25, 750-756.

Behura, J., M. Batzle, and R. Hofmann, 2006, Shear properties of oil shales, 76th Annual International Meeting, SEG, Expanded Abstracts, 1973-1977.

Caputo, M., 1967, Linear models of dissipation whose Q is almost frequency independent - II: Geophysics, 13, 529-539.

Cole, K., and R. Cole, 1941, Dispersion and absorption in dielectrics: Journal of Chemical Physics, 9, 341-351.

Davidson, D. W. and R. H. Cole, 1951, Dielectric relaxation in glycerol, propylene glycol and n-propanol: Journal of Chemical Physics, 19, 1484-1490.

De Ghetto, G., F. Paone, and M. Villa, 1995, Pressure-volume-temperature correlations for heavy and extra heavy oils: SPE 30316.

Han, D., J. Liu, and M. Batzle, 2005, Measurement of shear wave velocity of heavy oil: 75th Annual International Meeting, SEG, Expanded Abstracts, 1514-1517.

, 2006, Acoustic property of heavy oil — measured data: 76th Annual International Meeting, SEG, Expanded Abstracts, 1903-1907.

Haviliak, S. and S. Negami, 1967, A complex plane representation of dielectric and mechanical relaxation processes in some polymers: Polymer, 8, 161-210.

Kwan, K. S., 1998, The role of penetrant structure in the transport and mechanical properties of a thermoset adhesive (Chapter 6): Ph.D. dissertation. 